\title{
Adaptive Sliding Mode Control Based on Equivalence Principle and Its Application to Chaos Control in a Seven-Dimensional Power System
}

\author{
Jiangbin Wang $\mathbb{D}^{1},{ }^{1}$ Ling Liu $\mathbb{D},{ }^{1}$ Chongxin Liu, ${ }^{1}$ and Xiaoteng $\operatorname{Li} \mathbb{D}^{2}$ \\ ${ }^{1}$ State Key Laboratory of Electrical Insulation and Power Equipment, School of Electrical Engineering, Xi'an Jiaotong University, \\ Xi'an 710049, China \\ ${ }^{2}$ Electric Power Research Institute of State Grid Shaanxi Electric Power Company, Xi'an 710010, China
}

Correspondence should be addressed to Jiangbin Wang; 1550151867@qq.com

Received 12 November 2019; Revised 17 December 2019; Accepted 30 December 2019; Published 1 February 2020

Academic Editor: Mauro Gaggero

Copyright $(2020$ Jiangbin Wang et al. This is an open access article distributed under the Creative Commons Attribution License, which permits unrestricted use, distribution, and reproduction in any medium, provided the original work is properly cited.

The main purpose of the paper is to control chaotic oscillation in a complex seven-dimensional power system model. Firstly, in view that there are many assumptions in the design process of existing adaptive controllers, an adaptive sliding mode control scheme is proposed for the controlled system based on equivalence principle by combining fixed-time control and adaptive control with sliding mode control. The prominent advantage of the proposed adaptive sliding mode control scheme lies in that its design process breaks through many existing assumption conditions. Then, chaotic oscillation behavior of a seven-dimensional power system is analyzed by using bifurcation and phase diagrams, and the proposed strategy is adopted to control chaotic oscillation in the power system. Finally, the effectiveness and robustness of the designed adaptive sliding mode chaos controllers are verified by simulation.

\section{Introduction}

Power system, one of the high-dimensional and strongly coupled nonlinear systems, often exhibits complex nonlinear dynamic behaviors. Chaotic oscillation is an important phenomenon that has attracted considerable attention in recent years [1-3]. Results show that parameter variations, energy disturbance, and time delay can induce chaos in the power system [4-6]. Since chaotic oscillation affects its stability and may cause voltage collapse and other unacceptable accidents $[7,8]$, then how to effectively control chaos in the power system has become a common problem to be considered both theoretically and practically.

In recent years, various nonlinear control strategies have been applied to chaos control in power systems, such as feedback control [9], passive control [10], time-delay feedback control [9], quasi-sliding mode control based on relay characteristic function [11], variable speed synergetic control [12], and adaptive fixed-time fast terminal sliding mode control [13]. However, all aforementioned methods are aimed at a simple two-dimensional power system model. Some scholars have also designed chaos controllers for a classical four-dimensional power system model. At present, the main control methods proposed for the model include finite-time feedback control [14], finite-time integral sliding mode control [15], finite-time passive control [16], chattering-free time scale separation sliding mode control [17], fixed-time integral sliding mode control [18], feedback linearization based sliding mode control [19], discrete time sliding mode control [20], fractional order sliding mode control [21], fast fixed-time nonsingular terminal sliding mode control [22], and fixed-time dynamic surface highorder sliding mode control [23]. However, it is worth noting that most of these controllers are abstract control inputs without considering realizability of the controller and there are also too many control inputs (see [14-21]), which make the proposed control methods impractical.

Actually, due to the complexity of the power system model itself, studying chaos control in a more complex model has more important research value. Among many 
existing power system dynamic models, the seven-dimensional power system is the most complex model of all known power system models. Bifurcation and chaos phenomena in the model are analyzed in detail in [24]. Subsequently, several adverse effects of chaos on power system stability are studied based on the model in $[25,26]$. Corresponding to analysis of the model, controlling its chaos still remains an important research topic. However, there is still scarce literature reporting chaos control in such a complex model.

Among many nonlinear control methods, sliding mode control has attracted many scholars' attention because of its simplicity, reliability, and effectiveness. However, the designed control inputs depend on system functions according to the conventional sliding mode control method. In order to simplify the control input, adaptive sliding mode control can be designed to make its control inputs easy to be realized in engineering. However, as long as designing an adaptive controller, many assumptions will be introduced in the controller design process. The most common assumptions are the Lipschitz assumption and the bounded state variables assumption [27-32]. Specifically, the famous Lipschitz assumption is often introduced into the adaptive controllers designed in [27-30], and it actually limits the applicability of designed controllers. In addition, it is always impossible to verify the validity of the assumption condition for complex system models. However, the adaptive controller in [31, 32] introduces assumption that state variables of a controlled system are bounded. Although the assumption is valid for chaotic systems, the application scope of the designed controller is always limited as long as it is introduced. More importantly, it is difficult to apply these control methods to control complex systems such as the seven-dimensional power system due to the introduction of these assumptions. In addition, adaptive sliding mode controllers designed in $[33,34]$ will behave chattering.

To solve the above problems comprehensively, an adaptive sliding mode control scheme is proposed to control chaos in the seven-dimensional power system. First, in order to ensure that the designed adaptive sliding mode controller has better control effect, a fixed-time sliding mode controller is designed for a controlled system. Based on equivalence principle of control effect, an adaptive sliding mode control scheme is proposed. The unique advantage of the adaptive sliding mode control is that no assumptions are introduced in the controller design process. Then, the proposed control scheme is used to control chaotic oscillation in a sevendimensional power system. Simulation results verify its effectiveness and robustness.

The main contributions of this paper are as follows: (1) Compared with other adaptive sliding mode control methods, the proposed adaptive sliding mode controller based on the equivalence principle does not introduce any assumptions, and the designed control inputs does not include system functions, which make the designed control inputs to be directly applied to such complex dynamic system as the seven-dimensional power system model. (2) Compared with other power system chaos control methods, different from the existing methods which only control a simple two-dimensional and four-dimensional chaotic power system, this paper directly control the most complex seven-dimensional chaotic power system, and the designed controller is more universal. (3) Compared with many control methods which only design abstract control inputs, this paper introduces an active energy storage device and static var compensation device commonly used in power system and designs control inputs for two devices, which make the proposed control method to have certain engineering guidance.

The rest of the paper is organized as follows. The design process of the proposed adaptive sliding mode controller is given in Section 2. In Section 3, the proposed adaptive sliding mode control scheme is used to control chaotic oscillation in the seven-dimensional power system. In Section 4, the effectiveness of proposed adaptive sliding mode control method is verified by simulation. The conclusion is finally drawn in Section 5.

\section{Adaptive Sliding Mode Control}

\subsection{Mathematical Basis}

Definition 1 (see [35]). Consider a differential equation system:

$$
\begin{aligned}
\dot{x} & =f(x), \\
x(0) & =x_{0},
\end{aligned}
$$

where $x \in R$ and $f(x)$ denotes any nonlinear function.

Let the time required for the state variable of system (1) to stabilize to the origin be $T\left(x_{0}\right)$; if for $\forall x_{0} \in R, \exists T_{\max }>0$, such that $\lim _{x_{0}} \rightarrow \infty\left[T\left(x_{0}\right)\right] \leq T_{\max }$ holds, then the state variable of system (1) is said to be stable in a fixed time.

Lemma 1 (see [35]). Consider a continuous positive definite function $V$; let it satisfy differential inequality as follows:

$$
\dot{V} \leq-\left(\alpha V^{p}+\beta V^{q}\right)^{k}, \quad V(0)=V_{0},
$$

where $\alpha>0, \beta>0, p>0, q>0$, and $k>0$, and parameters $p, q$, and $k$ satisfy $p k<1$ and $q k>1$.

Let the time required for state variable $V$ of system (2) converging to the origin be $T\left(V_{0}\right)$; then, $V$ will converge to the origin in a fixed time upper bounded by a constant, that is, $\lim _{V_{0}} \longrightarrow \infty\left[T(V)_{0}\right] \leq T_{\max (V)}$, and

$$
T_{\max (V)}=\frac{1}{\alpha^{k}(1-p k)}+\frac{1}{\beta^{k}(q k-1)} \text {. }
$$

Lemma 2. Consider a differential equation system:

$$
\dot{y}=-\gamma\left(\alpha_{1}|y|^{m_{1} / n_{1}}+\beta_{1}|y|^{m_{2} / n_{2}}\right)^{m_{3} / n_{3}} \cdot \operatorname{sign}(y),
$$

where $\alpha_{1}>0, \beta_{1}>0$, and $m_{1}, n_{1}, m_{2}, n_{2}, m_{3}$, and $n_{3}$ satisfy $\left(m_{1} / n_{1}\right) \cdot\left(m_{3} / n_{3}\right)<1$ and $\left(m_{2} / n_{2}\right) \cdot\left(m_{3} / n_{3}\right)>1, \gamma$ is a constant coefficient, and $\gamma>0$.

Let the time required for the state variables of system (4) converging to the origin be $T\left(y_{0}\right)$; then, state variable $y$ will converge to the origin in a fixed time upper bounded by a constant $T_{\max (y)}$, that is, $\lim _{y_{0} \rightarrow \infty}\left[T\left(y_{0}\right)\right] \leq T_{\max (y)}$, and 


$$
\begin{aligned}
T_{\max (y)}= & \frac{1}{\gamma \cdot \alpha_{1}^{m_{3} / n_{3}}\left[1-\left(m_{1} / n_{1}\right)\left(m_{3} / n_{3}\right)\right]} \\
& +\frac{1}{\gamma \cdot \beta_{1}^{m_{3} / n_{3}}\left[\left(m_{2} / n_{2}\right)\left(m_{3} / n_{3}\right)-1\right]} .
\end{aligned}
$$

Proof. Construct a Lyapunov function $W=|y|$; then, its derivative with respect to time is

$$
\begin{aligned}
\dot{W} & =\dot{y} \cdot \operatorname{sign}(y)=-\gamma\left(\alpha_{1}|y|^{m_{1} / n_{1}}+\beta_{1}|y|^{m_{2} / n_{2}}\right)^{m_{3} / n_{3}} \\
& =-\gamma\left(\alpha_{1} W^{m_{1} / n_{1}}+\beta_{1} W^{m_{2} / n_{2}}\right)^{m_{3} / n_{3}} \\
& =-\left(\gamma^{n_{3} / m_{3}}\right)^{m_{3} / n_{3}}\left(\alpha_{1} W^{m_{1} / n_{1}}+\beta_{1} W^{m_{2} / n_{2}}\right)^{m_{3} / n_{3}} \\
& =-\left(\gamma^{n_{3} / m_{3}} \cdot \alpha_{1} W^{m_{1} / n_{1}}+\gamma^{n_{3} / m_{3}} \cdot \beta_{1} W^{m_{2} / n_{2}}\right)^{m_{3} / n_{3}} .
\end{aligned}
$$

Let the time required for the state variable $W$ converging to the origin be $T\left(W_{0}\right)$; from Lemma 1 , we can obtain that $\lim _{W_{0} \longrightarrow \infty}\left[T(W)_{0}\right] \leq T_{\max (W)}$ and

$$
\begin{aligned}
T_{\max (W)}= & \frac{1}{\left(\gamma^{n_{3} / m_{3}} \cdot \alpha_{1}\right)^{m_{3} / n_{3}}\left(1-m_{1} m_{3} / n_{1} n_{3}\right)} \\
& +\frac{1}{\left(\gamma^{n_{3} / m_{3}} \cdot \beta_{1}\right)^{m_{3} / n_{3}}\left(m_{2} m_{3} / n_{2} n_{3}-1\right)}=T_{\max (y)} .
\end{aligned}
$$

The proof is completed.

2.2. Controller Design. Consider a controlled nonlinear system as follows:

$$
\dot{x}_{i}=f_{i}(x, t)+g_{i}(x) \cdot u_{i},
$$

where $x=\left[x_{1}, x_{2}, \ldots, x_{N}\right]^{T} \in R^{N}$ represents state vector and $x_{i} \in x, i=1,2, \ldots, N ; f_{i}(x, t) \in R$ denotes nonlinear system function of $x$ and $t ; g_{i}(x) \in R$ represents control input gain, and $g_{i}(x) \neq 0 ; u_{i}$ represents control input.

Let the control objective of system (8) be a constant value $x_{i d}$; then, control error can be written as $e_{i}=x_{i}-x_{i d}$, and one can get from system (8) that its error dynamics is

$$
\dot{e}_{i}=\dot{x}_{i}-\dot{x}_{i d}=\dot{x}_{i}=f_{i}(x, t)+g_{i}(x) \cdot u_{i} .
$$

In order to ensure better control effect of the designed controller, a fixed-time control input which can make the state variable $e_{i}$ converge precisely to zero is designed for controlled system (9).

For the error system (9), an integral fixed-time sliding surface is designed as

$$
s_{i}=e_{i}+\gamma_{i} \int_{0}^{t}\left[\left(\alpha_{1}\left|e_{i}\right|^{m_{1} / n_{1}}+\beta_{1}\left|e_{i}\right|^{m_{2} / n_{2}}\right)^{m_{3} / n_{3}} \cdot \operatorname{sign}\left(e_{i}\right)\right] \mathrm{d} t,
$$

where $\gamma_{i}>0, \alpha_{1}>0$, and $\beta_{1}>0$.

To make system state reach the sliding surface $s_{i}=0$ in a fixed time, let

$$
\begin{aligned}
\dot{s}_{i}= & \dot{e}_{i}+\gamma_{i}\left(\alpha_{1}\left|e_{i}\right|^{m_{1} / n_{1}}+\beta_{1}\left|e_{i}\right|^{m_{2} / n_{2}}\right)^{m_{3} / n_{3}} \cdot \operatorname{sign}\left(e_{i}\right) \\
= & f_{i}(x, t)+g_{i}(x) u_{i}+\gamma_{i}\left(\alpha_{1}\left|e_{i}\right|^{m_{1} / n_{1}}+\beta_{1}\left|e_{i}\right|^{m_{2} / n_{2}}\right)^{m_{3} / n_{3}} \\
& \cdot \operatorname{sign}\left(e_{i}\right) \\
= & -\varepsilon_{i}\left(\alpha_{2}\left|s_{i}\right|^{m_{1} / n_{1}}+\beta_{2}\left|s_{i}\right|^{m_{2} / n_{2}}\right)^{m_{3} / n_{3}} \cdot \operatorname{sign}\left(s_{i}\right),
\end{aligned}
$$

where $\varepsilon_{i}>0, \alpha_{2}>0$, and $\beta_{2}>0$.

According to Lemma 2 , if the control input $u_{i}$ is designed according to equation (11), the designed control input can make the system state reach the sliding surface $s_{i}=0$ in a fixed time; then, $\dot{s}_{i}=0$, namely, $\dot{e}_{i}=-\gamma_{i}\left(\alpha_{1}\left|e_{i}\right|^{m_{1} / n_{1}}\right.$ $\left.+\beta_{1}\left|e_{i}\right|^{m_{2} / n_{2}}\right)^{m_{3} / n_{3}} \operatorname{sign}\left(e_{i}\right)$; this shows that when the system state reaches the sliding surface, the error $e_{i}$ will also converge to zero in a fixed time, thus completing the control of system (8).

From equation (11), the expression of controller $u_{i(\mathrm{ref})}$ $=u_{i}$ can be solved as follows:

$$
\begin{aligned}
u_{i(\mathrm{ref})}= & -\frac{1}{g_{i}(x)}\left[f_{i}(x, t)+\gamma_{i}\left(\alpha_{1}\left|e_{i}\right|^{m_{1} / n_{1}}+\beta_{1}\left|e_{i}\right|^{m_{2} / n_{2}}\right)^{m_{3} / n_{3}}\right. \\
& \left.\cdot \operatorname{sign}\left(e_{i}\right)+\varepsilon_{i}\left(\alpha_{2}\left|s_{i}\right|^{m_{1} / n_{1}}+\beta_{2}\left|s_{i}\right|^{m_{2} / n_{2}}\right)^{m_{3} / n_{3}} \cdot \operatorname{sign}\left(s_{i}\right)\right] .
\end{aligned}
$$

To design an adaptive sliding mode control input for system (8), control input in (12) can be used as a reference and design real control input $u_{i \text { (real) }}=u_{i}$ for system (8) as

$$
\begin{aligned}
u_{i(\text { real })}= & -\frac{1}{g_{i}(x)}\left[\widehat{\gamma}_{i}\left(\alpha_{1}\left|e_{i}\right|^{m_{1} / n_{1}}+\beta_{1}\left|e_{i}\right|^{m_{2} / n_{2}}\right)^{m_{3} / n_{3}} \operatorname{sign}\left(e_{i}\right)\right. \\
& \left.+\varepsilon_{i}\left(\alpha_{2}\left|s_{i}\right|^{m_{1} / n_{1}}+\beta_{2}\left|s_{i}\right|^{m_{2} / n_{2}}\right)^{m_{3} / n_{3}} \cdot \operatorname{sign}\left(s_{i}\right)\right],
\end{aligned}
$$

where $\hat{\gamma}_{i}$ denotes the adaptive parameters.

The design principle of adaptive law for adaptive parameter $\hat{\gamma}_{i}$ is to make real input $u_{i \text { (real) }}$ and reference input $u_{i(\text { ref })}$ equivalent to each other after adaptive parameter $\hat{\gamma}_{i}$ converges to a constant value $\widehat{\gamma}_{i}^{*}$, that is, to make $u_{i \text { (real) }}$ and $u_{i(\text { ref })}$ satisfy the equality constraint:

$$
\begin{aligned}
u_{i(\text { real })}^{*}= & -\frac{1}{g_{i}(x)}\left[\widehat{\gamma}_{i}^{*}\left(\alpha_{1}\left|e_{i}\right|^{m_{1} / n_{1}}+\beta_{1}\left|e_{i}\right|^{m_{2} / n_{2}}\right)^{m_{3} / n_{3}} \cdot \operatorname{sign}\left(e_{i}\right)\right. \\
& \left.+\varepsilon_{i}\left(\alpha_{2}\left|s_{i}\right|^{m_{1} / n_{1}}+\beta_{2}\left|s_{i}\right|^{m_{2} / n_{2}}\right)^{m_{3} / n_{3}} \cdot \operatorname{sign}\left(s_{i}\right)\right]=u_{i(\text { ref })},
\end{aligned}
$$

where $\widehat{\gamma}_{i}^{*}$ represents the constant value after adjustment of adaptive parameter $\hat{\gamma}_{i}$.

When the real control input of system (9) is designed as in (13) and taking constraint (14) into account, then system (9) can be rewritten as 


$$
\begin{aligned}
\dot{e}_{i}= & f_{i}(x, t)-\left[\widehat{\gamma}_{i}\left(\alpha_{1}\left|e_{i}\right|^{m_{1} / n_{1}}+\beta_{1}\left|e_{i}\right|^{m_{2} / n_{2}}\right)^{m_{3} / n_{3}} \cdot \operatorname{sign}\left(e_{i}\right)\right. \\
& \left.+\varepsilon_{i}\left(\alpha_{2}\left|s_{i}\right|^{m_{1} / n_{1}}+\beta_{2}\left|s_{i}\right|^{m_{2} / n_{2}}\right)^{m_{3} / n_{3}} \cdot \operatorname{sign}\left(s_{i}\right)\right] \\
= & f_{i}(x, t)-\left[\widehat{\gamma}_{i}\left(\alpha_{1}\left|e_{i}\right|^{m_{1} / n_{1}}+\beta_{1}\left|e_{i}\right|^{m_{2} / n_{2}}\right)^{m_{3} / n_{3}} \cdot \operatorname{sign}\left(e_{i}\right)\right. \\
& \left.+\varepsilon_{i}\left(\alpha_{2}\left|s_{i}\right|^{m_{1} / n_{1}}+\beta_{2}\left|s_{i}\right|^{m_{2} / n_{2}}\right)^{m_{3} / n_{3}} \cdot \operatorname{sign}\left(s_{i}\right)\right]+g_{i}(x) u_{i(\text { ref })} \\
& -g_{i}(x) u_{i(\text { real })}^{*}
\end{aligned}
$$

By substituting (12) and (14) into (15), we can obtain

$$
\begin{aligned}
\dot{e}_{i}= & \left(\widehat{\gamma}_{i}^{*}-\widehat{\gamma}_{i}-\gamma_{i}\right)\left[\left(\alpha_{1}\left|e_{i}\right|^{m_{1} / n_{1}}+\beta_{1}\left|e_{i}\right|^{m_{2} / n_{2}}\right)^{m_{3} / n_{3}} \cdot \operatorname{sign}\left(e_{i}\right)\right] \\
& -\varepsilon_{i}\left(\alpha_{2}\left|s_{i}\right|^{m_{1} / n_{1}}+\beta_{2}\left|s_{i}\right|^{m_{2} / n_{2}}\right)^{m_{3} / n_{3}} \cdot \operatorname{sign}\left(s_{i}\right) .
\end{aligned}
$$

To design the adaptive law for adaptive parameter $\widehat{\gamma}_{i}$, a Lyapunov function is constructed as

$$
V_{1}=\sum_{i=1}^{N}\left[\frac{1}{2} s_{i}^{2}+\frac{1}{2}\left(\widehat{\gamma}_{i}-\widehat{\gamma}_{i}^{*}\right)^{2}\right] \text {. }
$$

Then, its derivative with respect to time is

$$
\begin{aligned}
\dot{V}_{1}= & \sum_{i=1}^{N}\left[s_{i} \dot{s}_{i}+\left(\widehat{\gamma}_{i}-\widehat{\gamma}_{i}^{*}\right) \dot{\hat{\gamma}}_{i}\right] \\
= & \sum_{i=1}^{N}\left\{s_{i}\left[\dot{e}_{i}+\gamma_{i}\left(\alpha_{1}\left|e_{i}\right|^{m_{1} / n_{1}}+\beta_{1}\left|e_{i}\right|^{m_{2} / n_{2}}\right)^{m_{3} / n_{3}} \cdot \operatorname{sign}\left(e_{i}\right)\right]\right. \\
& \left.+\left(\hat{\gamma}_{i}-\hat{\gamma}_{i}^{*}\right) \dot{\hat{\gamma}}_{i}\right\} .
\end{aligned}
$$

By substituting (16) into (18), one can obtain

$$
\begin{aligned}
\dot{V}_{1}= & \sum_{i=1}^{N}\left[s_{i} \dot{s}_{i}+\left(\widehat{\gamma}_{i}-\widehat{\gamma}_{i}^{*}\right) \dot{\hat{\gamma}}_{i}\right] \\
= & \sum_{i=1}^{N}\left[s_{i} \cdot\left(\widehat{\gamma}_{i}^{*}-\widehat{\gamma}_{i}-\gamma_{i}\right)\left(\alpha_{1}\left|e_{i}\right|^{m_{1} / n_{1}}+\beta_{1}\left|e_{i}\right|^{m_{2} / n_{2}}\right)^{m_{3} / n_{3}}\right. \\
& \left.\cdot \operatorname{sign}\left(e_{i}\right)\right]-\sum_{i=1}^{N}\left[s_{i} \cdot \varepsilon_{i}\left(\alpha_{2}\left|s_{i}\right|^{m_{1} / n_{1}}+\beta_{2}\left|s_{i}\right|^{m_{2} / n_{2}}\right)^{m_{3} / n_{3}}\right. \\
& \left.\cdot \operatorname{sign}\left(s_{i}\right)\right]+\sum_{i=1}^{N}\left[s_{i} \cdot \gamma_{i}\left(\alpha_{1}\left|e_{i}\right|^{m_{1} / n_{1}}+\beta_{1}\left|e_{i}\right|^{m_{2} / n_{2}}\right)^{m_{3} / n_{3}}\right. \\
& \left.\cdot \operatorname{sign}\left(e_{i}\right)+\left(\widehat{\gamma}_{i}-\widehat{\gamma}_{i}^{*}\right) \dot{\hat{\gamma}}_{i}\right] .
\end{aligned}
$$

Design the adaptive law as

$$
\dot{\hat{\gamma}}_{i}=\left(\alpha_{1}\left|e_{i}\right|^{m_{1} / n_{1}}+\beta_{1}\left|e_{i}\right|^{m_{2} / n_{2}}\right)^{m_{3} / n_{3}} \cdot \operatorname{sign}\left(e_{i}\right) \cdot s_{i} \text {. }
$$

Then, we can know that

$$
\dot{V}_{1}=\sum_{i=1}^{N}\left[-\varepsilon_{i}\left(\alpha_{2}\left|s_{i}\right|^{m_{1} / n_{1}}+\beta_{2}\left|s_{i}\right|^{m_{2} / n_{2}}\right)^{m_{3} / n_{3}} \cdot\left|s_{i}\right|\right] \leq 0 .
$$

It means from $\dot{V}_{1} \leq 0$ that the system state finally reaches the sliding surface $s_{i}=0$, and the adaptive parameter $\widehat{\gamma}_{i}$ converges to a constant $\widehat{\gamma}_{i}^{*}$; then, the error variable $e_{i}$ converges to zero, thus completing control of system (8).

The above controller design process shows that as long as control input of system (8) is finally designed as an adaptive sliding mode controller (22), then system (8) will be controlled to the objective:

$$
\left\{\begin{array}{l}
u_{i \text { (real })}=-\left(\frac{1}{g_{i}(x)}\right) \cdot\left[\widehat{\gamma}_{i}\left(\alpha_{1}\left|e_{i}\right|^{m_{1} / n_{1}}+\beta_{1}\left|e_{i}\right|^{m_{2} / n_{2}}\right)^{m_{3} / n_{3}} \cdot \operatorname{sign}\left(e_{i}\right)+\varepsilon_{i}\left(\alpha_{2}\left|s_{i}\right|^{m_{1} / n_{1}}+\beta_{2}\left|s_{i}\right|^{m_{2} / n_{2}}\right)^{m_{3} / n_{3}} \cdot \operatorname{sign}\left(s_{i}\right)\right] \\
\dot{\hat{\gamma}}_{i}=\left(\alpha_{1}\left|e_{i}\right|^{m_{1} / n_{1}}+\beta_{1}\left|e_{i}\right|^{m_{2} / n_{2}}\right)^{m_{3} / n_{3}} \cdot \operatorname{sign}\left(e_{i}\right) \cdot s_{i}
\end{array}\right.
$$

where $s_{i}=e_{i}+\gamma_{i} \int_{0}^{t}\left[\left(\alpha_{1}\left|e_{i}\right|^{m_{1} / n_{1}}+\beta_{1}\left|e_{i}\right|^{m_{2} / n_{2}}\right)^{m_{3} / n_{3}} \operatorname{sign}\left(e_{i}\right)\right] \mathrm{d} t$.

Remark 1. Problems studied in [27-30] can be summed up as designing an adaptive controller for controlled system (8). However, these methods introduce Lipschitz assumption in the design process and can only control the state variable to the corresponding equilibrium point, because their stability proof processes need to satisfy $f_{i}\left(x_{0}\right)=0$, where $f_{i}\left(x_{0}\right)$ denotes the controlled system function and $x_{0}$ represents the control objective. While it is worth noticing that the proposed controller design process does not introduce any assumptions and any constant objective can be realized for controlled system (8) by the designed controller.
Remark 2. Problems studied in $[31,32]$ can also be attributed to designing an adaptive controller for controlled system (8). However, these methods introduce assumption that state variable $x_{i}$ of controlled system (8) is bounded in the controller design process, i.e., the assumption that $\left|x_{i}\right| \leq M$, where $M$ is a constant.

Remark 3. Adaptive sliding mode controllers designed in $[33,34]$ behave obvious chattering, which makes it difficult to apply in practice. However, the proposed control inputs are continuous and there is no chattering phenomenon. 
Remark 4. If disturbances and uncertainties are not considered, problems studied in [36-38] can be reduced to designing sliding mode controllers for controlled systems (8), while their control inputs include system function $f_{i}(x, t)$. Since it is often difficult to obtain the exact system model in practical, then these controller design methods greatly reduce their practicability. However, the proposed controller does not contain $f_{i}(x, t)$, and it can be applied to control complex systems such as seven-dimensional power system.

\section{Adaptive Sliding Mode Control for Chaotic Oscillation in Power Systems}

3.1. System Model and Its Chaotic Oscillation. The sevendimensional power system model is a most complex one among many power system models, and its dynamics can be described as [24-26]

$$
\left\{\begin{array}{l}
\dot{\delta}_{\mathrm{m}}=\omega_{\mathrm{B}} s_{\mathrm{m}}, \\
\dot{s}_{\mathrm{m}}=\frac{-d s_{\mathrm{m}}+P_{\mathrm{m}}-P_{\mathrm{g}}}{2 H}, \\
\dot{E}_{q}^{\prime}=\frac{-E_{q}^{\prime}+\left(x_{d}-x_{d}^{\prime}\right) I_{d}+E_{\mathrm{fd}}}{T_{d 0}}, \\
\dot{E}_{d}^{\prime}=\frac{-E_{d}^{\prime}-\left(x_{q}-x_{q}^{\prime}\right) I_{q}}{T_{q 0}}, \\
\dot{E}_{\mathrm{fd}}=\frac{-E_{\mathrm{fd}}+K_{\mathrm{A}}\left(V_{\mathrm{ref}}-V\right)_{\mathrm{t}}}{T_{\mathrm{A}}}, \\
\dot{\delta}_{\mathrm{L}}=\frac{\mathrm{Q}-Q_{1 \mathrm{~d}}-Q_{0}-q_{2} V_{\mathrm{L}}-\left(q_{3}-B_{\mathrm{c}}\right) V_{\mathrm{L}}^{2}}{q_{1}}, \\
\dot{V}_{\mathrm{L}}=\frac{P-P_{1 \mathrm{~d}}-P_{0}-p_{1} \cdot f(\cdot) / q_{1}-p_{3} V_{\mathrm{L}}}{p_{2}},
\end{array}\right.
$$

where $\delta_{\mathrm{m}}$ and $s_{\mathrm{m}}$ represent generator angle and generator slip, respectively; $E_{q}^{\prime}$ and $E_{d}^{\prime}$ represent transient potential of damper winding on $\mathrm{q}$-axis and $\mathrm{d}$-axis, respectively; $E_{\mathrm{fd}}$ denotes the excitation potential; $\delta_{\mathrm{L}}$ and $V_{\mathrm{L}}$ represent voltage phase angle and amplitude of the load bus, respectively; and $f(\cdot)=Q-Q_{1 d}-Q_{0}-q_{2} V_{L}-\left(q_{3}-B_{c}\right) V_{L}^{2}$.

Parameter coupling relationships and parameter values of system (23) are shown in the Appendix. The initial values of the power system are selected as follows: $\delta_{\mathrm{m}}(0)=1.3331$, $s_{\mathrm{m}}(0)=0, \quad E_{q}^{\prime}(0)=1.332678, \quad E_{d}^{\prime}(0)=-0.3283$, $E_{\mathrm{fd}}(0)=4.198, \delta_{\mathrm{L}}(0)=0.2396$, and $V_{\mathrm{L}}(0)=0.93$. Among many system parameters, $P_{\mathrm{m}}$ is an important bifurcation parameter to find the parameter condition for chaotic oscillation. Under the parameters given in the Appendix, and when the variation range of $P_{\mathrm{m}}$ is $P_{\mathrm{m}} \in[1.34,1.363]$, the maximum method can be used to draw the bifurcation diagram of system state $\delta_{\mathrm{m}}$ varying with parameter $P_{\mathrm{m}}$ as shown in Figure 1 .

From the bifurcation diagram, it can be concluded that when $P_{\mathrm{m}} \in[1.34,1.3552]$, the system is under periodic motion state. When $P_{\mathrm{m}} \in[1.3552,1.3605]$, typical period doubling bifurcations occur in the system. And in the interval $P_{\mathrm{m}} \in[1.3605,1.363]$, the whole system enters into chaotic state completely, and chaotic oscillation occurs in the power system. In order to obtain the phase diagram of chaotic oscillation, let $P_{\mathrm{m}}=1.363$, then the system phase diagram can be obtained as shown in Figure 2. System state behaves as a strange attractor on phase diagram, and the time responses of state variables must behave as aperiodic waveforms, which are not allowed in the stable operation of the power system. Therefore, the chaos controller must be designed for chaotic power systems.

3.2. Controller Design for Chaos in Power System. The sevendimensional power system is a typical three-bus power system. The three buses include generator bus, load bus, and infinite bus. To control chaotic oscillation and restore the whole system from chaotic oscillation to stable operation, it is necessary to restore the whole system to re-synchronization and control the load bus voltage to its rated value. In this way, the chaos control problem in power systems comes down to the control of active power and reactive power of the whole system. In order to make the designed controllers have a certain engineering practical value, we connect the active energy storage device on the generator bus and static var compensation device on the load bus and introduce dynamic models of two devices [39-41]; then, the controlled power system model can be given by combining dynamics of these devices with power system model as

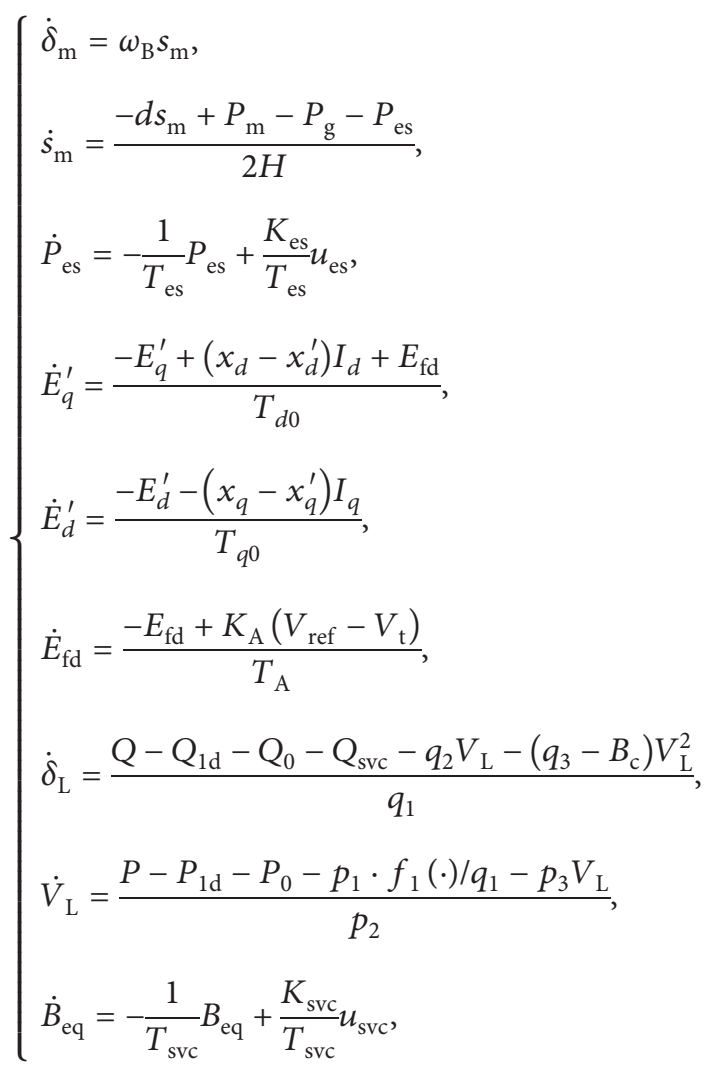

where $P_{\text {es }}$ represents the active power absorbed by energy storage device; $T_{\mathrm{es}}, K_{\mathrm{es}}$, and $u_{\mathrm{es}}$ represent the time constant, 


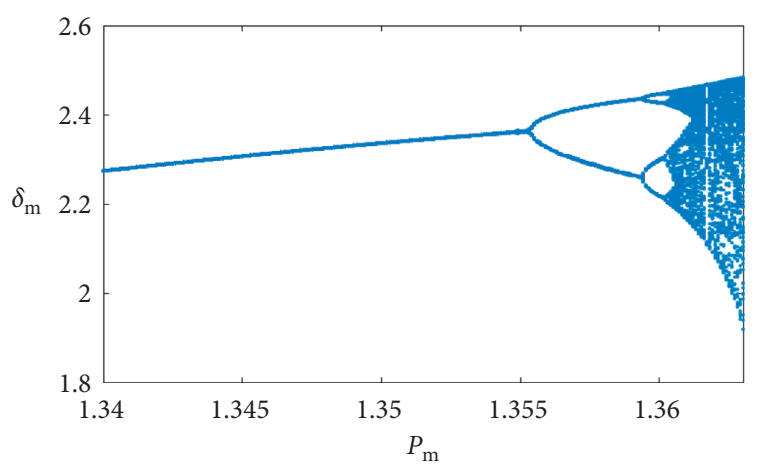

Figure 1: Bifurcation diagram of system state $\delta_{\mathrm{m}}$ varying with $P_{\mathrm{m}}$.

control input gain, and control input of the energy storage device, respectively; $B_{\mathrm{eq}}$ and $T_{\mathrm{svc}}$ represent the equivalent admittance and time constant of static var compensator; $K_{\mathrm{svc}}$ and $u_{\mathrm{svc}}$ represent the control input gain and control input of static var compensator; $Q_{\mathrm{svc}}=B_{\mathrm{eq}} V_{\mathrm{L}}^{2}$, which represents the reactive power supplied by static var compensator to load bus; and $f_{1}(\cdot)=Q-Q_{1 d}-Q_{0}-Q_{s v c}-q_{2} V_{L}-\left(q_{3}-B_{c}\right) V_{\mathrm{L}}^{2}$.

To achieve the control objective for system (24), its output is determined as follows: $y_{1}=\delta_{\mathrm{m}}$ and $y_{2}=V_{\mathrm{L}}$. Then, sliding mode functions are designed as

$$
\begin{cases}\sigma_{1}=\left(\frac{\mathrm{d}}{\mathrm{d} t}+\lambda_{1}\right)^{2} \delta_{\mathrm{m}}, & \lambda_{1}>0 \\ \sigma_{2}=\left(\frac{\mathrm{d}}{\mathrm{d} t}+\lambda_{2}\right)\left(V_{\mathrm{L}}-1\right), & \lambda_{2}>0\end{cases}
$$

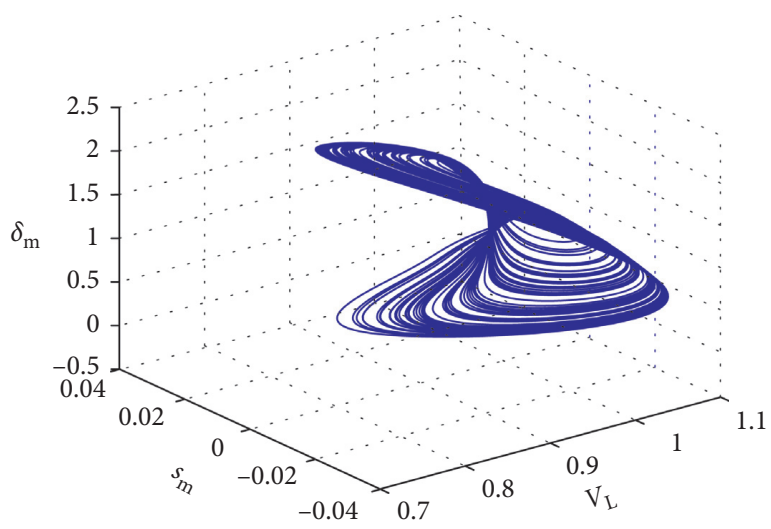

FIgURE 2: Phase diagram of the seven-dimensional chaotic power system.

It can be concluded from (25) that the derivative of sliding mode function $\sigma_{1}$ with respect to time is

$$
\dot{\sigma}_{1}=\dddot{\delta}_{\mathrm{m}}+2 \lambda_{1} \ddot{\delta}_{\mathrm{m}}+\lambda_{1}^{2} \dot{\delta}_{\mathrm{m}}=f_{\mathrm{es}}+g_{\mathrm{es}} u_{\mathrm{es}},
$$

where

$$
\left\{f_{\mathrm{es}}=\frac{\omega_{\mathrm{B}}}{2 H}\left(-d \dot{s}_{\mathrm{m}}-\dot{P}_{\mathrm{g}}+\frac{1}{T_{\mathrm{es}}} P_{\mathrm{es}}\right)+2 \lambda_{1} \ddot{\delta}_{\mathrm{m}}+\lambda_{1}^{2} \dot{\delta}_{\mathrm{m}}, g_{\mathrm{es}}=-\frac{\omega_{\mathrm{B}} K_{\mathrm{es}}}{2 H \cdot T_{\mathrm{es}}} .\right.
$$

It can also be concluded from (25) that the derivative of sliding mode function $\sigma_{2}$ with respect to time is

$$
\dot{\sigma}_{2}=\ddot{V}_{\mathrm{L}}+\lambda_{2} \dot{V}_{\mathrm{L}}=f_{\mathrm{svc}}+g_{\mathrm{svc}} u_{\mathrm{svc}} \text {, }
$$

where

$$
\left\{\begin{array}{l}
f_{\mathrm{svc}}=\frac{\dot{P}-p_{1}\left[\dot{Q}+B_{\mathrm{eq}} V_{\mathrm{L}}^{2} / T_{\mathrm{svc}}-B_{\mathrm{eq}} \cdot 2 V_{\mathrm{L}} \dot{V}_{\mathrm{L}}-q_{2} \dot{V}_{\mathrm{L}}-\left(q_{3}-B_{\mathrm{c}}\right) 2 V_{\mathrm{L}} \dot{V}_{\mathrm{L}}\right] / q_{1}-p_{3} \dot{V}_{\mathrm{L}}}{p_{2}}+\lambda_{2} \dot{V}_{\mathrm{L}} \\
g_{\mathrm{svc}}=\frac{p_{1} K_{\mathrm{svc}} V_{\mathrm{L}}^{2}}{q_{1} p_{2} T_{\mathrm{svc}}} .
\end{array}\right.
$$

The system composed of (26) and (28) is expressed as follows:

$$
\left\{\begin{array}{l}
\dot{\sigma}_{1}=f_{\mathrm{es}}+g_{\mathrm{es}} u_{\mathrm{es}} \\
\dot{\sigma}_{2}=f_{\mathrm{svc}}+g_{\mathrm{svc}} u_{\mathrm{svc}}
\end{array}\right.
$$

In this way, the chaos control problem of complex sevendimensional power system is finally transformed into the control problem of two first-order systems with sliding mode functions $\sigma_{1}$ and $\sigma_{2}$ as state variables given as in (30).
And control objective is to make $\sigma_{1}$ and $\sigma_{2}$ converge to 0 , that is, the state of controlled system (24) reaches sliding surfaces $\sigma_{1}=\left\{\delta_{\mathrm{m}} \mid \sigma_{1}\left(\delta_{\mathrm{m}}\right)=0\right\}$ and $\sigma_{2}=\left\{V_{\mathrm{L}} \mid \sigma_{2}\left(V_{\mathrm{L}}\right)=0\right\}$. Once the system state of the power system model reaches sliding surfaces, the control objective can be achieved from (25).

According to the proposed adaptive sliding mode control scheme, adaptive sliding mode controllers designed for system (30) (i.e., system (24)) can be expressed as follows: 


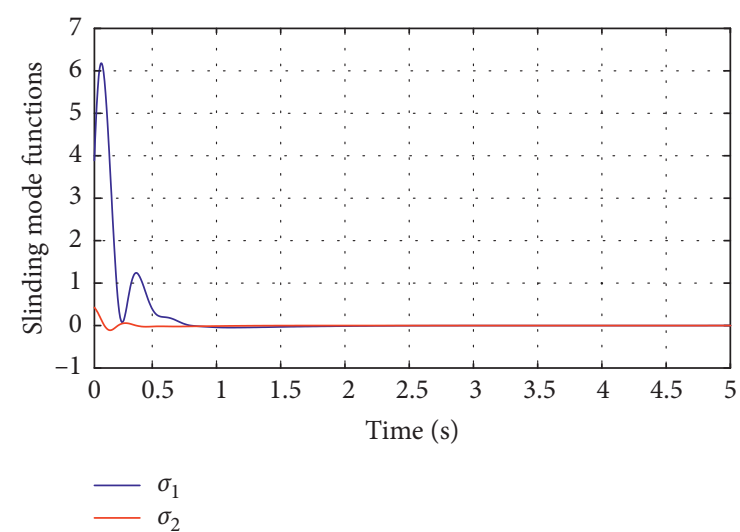

Figure 3: Time responses of sliding mode functions.

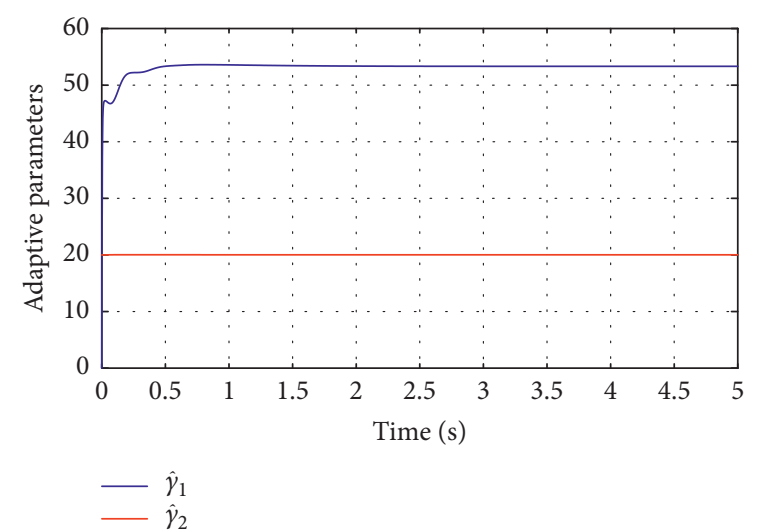

Figure 4: Adjustment curves of adaptive parameters.

$$
\left\{\begin{array}{l}
u_{\mathrm{es}}=-\frac{1}{g_{\mathrm{es}}}\left[\widehat{\gamma}_{1}\left(\alpha_{1}\left|\sigma_{1}\right|^{m_{1} / n_{1}}+\beta_{1}\left|\sigma_{1}\right|^{m_{2} / n_{2}}\right)^{m_{3} / n_{3}} \cdot \operatorname{sign}\left(\sigma_{1}\right)+\varepsilon_{1}\left(\alpha_{2}\left|s_{1}\right|^{m_{1} / n_{1}}+\beta_{2}\left|s_{1}\right|^{m_{2} / n_{2}}\right)^{m_{3} / n_{3}} \cdot \operatorname{sign}\left(s_{1}\right)\right], \\
\dot{\hat{\gamma}}_{1}=\left(\alpha_{1}\left|\sigma_{1}\right|^{m_{1} / n_{1}}+\beta_{1}\left|\sigma_{1}\right|^{m_{2} / n_{2}}\right)^{m_{3} / n_{3}} \cdot \operatorname{sign}\left(\sigma_{1}\right) \cdot s_{1}, \\
u_{\mathrm{svc}}=-\frac{1}{g_{\mathrm{svc}}}\left[\widehat{\gamma}_{2}\left(\alpha_{1}\left|\sigma_{2}\right|^{m_{1} / n_{1}}+\beta_{1}\left|\sigma_{2}\right|^{m_{2} / n_{2}}\right)^{m_{3} / n_{3}} \cdot \operatorname{sign}\left(\sigma_{2}\right)+\varepsilon_{2}\left(\alpha_{2}\left|s_{2}\right|^{m_{1} / n_{1}}+\beta_{2}\left|s_{2}\right|^{m_{2} / n_{2}}\right)^{m_{3} / n_{3}} \cdot \operatorname{sign}\left(s_{2}\right)\right], \\
\dot{\hat{\gamma}}_{2}=\left(\alpha_{1}\left|\sigma_{2}\right|^{m_{1} / n_{1}}+\beta_{1}\left|\sigma_{2}\right|^{m_{2} / n_{2}}\right)^{m_{3} / n_{3}} \cdot \operatorname{sign}\left(\sigma_{2}\right) \cdot s_{2},
\end{array}\right.
$$

where

$$
\left\{\begin{array}{r}
s_{1}=\sigma_{1}+\gamma_{1} \int_{0}^{t}\left[\left(\alpha_{1}\left|\sigma_{1}\right|^{m_{1} / n_{1}}+\beta_{1}\left|\sigma_{1}\right|^{m_{2} / n_{2}}\right)^{m_{3} / n_{3}} \cdot \operatorname{sign}\left(\sigma_{1}\right)\right] \mathrm{d} t, \\
s_{2}=\sigma_{2}+\gamma_{2} \int_{0}^{t}\left[\left(\alpha_{1}\left|\sigma_{2}\right|^{m_{1} / n_{1}}+\beta_{1}\left|\sigma_{2}\right|^{m_{2} / n_{2}}\right)^{m_{3} / n_{3}} \cdot \operatorname{sign}\left(\sigma_{2}\right)\right] \mathrm{d} t, \\
\gamma_{1}>0, \gamma_{2}>0, \varepsilon_{1}>0, \varepsilon_{2}>0 .
\end{array}\right.
$$

Remark 5. If the controllers for system (30) is designed according to the conventional controller design method, then the control inputs must contain complex system function terms $f_{\text {es }}$ and $f_{\text {svc }}$. However, since the adaptive sliding mode control input (31) is given for system (30), the terms $f_{\text {es }}$ and $f_{\text {svc }}$ will not appear in the control inputs.

\section{Simulation}

Example 1. First, the effectiveness of the proposed control method is verified. Control parameters are selected as follows: $K_{\mathrm{es}}=1, T_{\mathrm{es}}=1, K_{\mathrm{svc}}=1, T_{\mathrm{svc}}=1, \lambda_{1}=5, \lambda_{2}=5$, $\gamma_{1}=5, \quad \gamma_{2}=5, \varepsilon_{1}=10, \varepsilon_{2}=10, \alpha_{1}=1, \quad \beta_{1}=1, \alpha_{2}=1$, $\beta_{2}=1, m_{1}=7, n_{1}=5, m_{2}=7, n_{2}=3, m_{3}=3$, and $n_{3}=5$. It is worth noting that the random values of control parameters here are only to demonstrate the effectiveness of the proposed control method. In engineering practice, these values can be artificially adjusted according to the actual operating conditions of the system. The initial values of the adaptive parameters are $\hat{\gamma}_{1}(0)=0$ and $\widehat{\gamma}_{2}(0)=20$. After the designed two adaptive sliding mode controllers (31) are put into operation, the time response curves of sliding mode functions are shown in Figure 3. It is obvious that two sliding mode functions defined in (25) converge rapidly to 0 , which indicates that the state of controlled seven-dimensional power system (24) reaches sliding mode surfaces.

In the whole control process, the adaptive parameters are shown in Figure 4, and the two adaptive parameters are gradually adjusted to constant values. Figures 5 and 6 present the state variables of controlled power system (24). Each state variable restores to a stable state, and chaotic oscillation in the seven-dimensional power system is effectively suppressed. The evolution process diagram of the states of the uncontrolled and controlled system in phase space is shown in Figure 7. It can be seen that the state of uncontrolled power system eventually evolves into a strange attractor over time, while the state of controlled system evolves from the original chaotic attractor into a equilibrium fixed point in phase space 


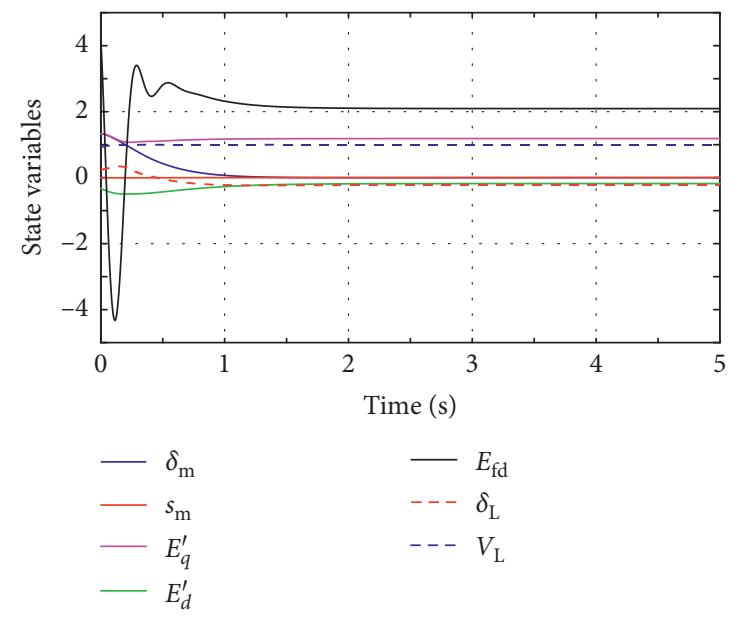

Figure 5: Time responses of controlled power system.

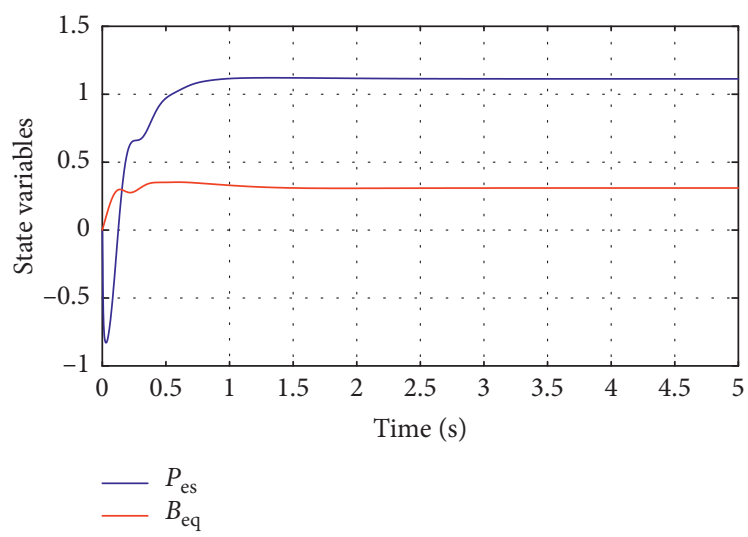

Figure 6: Active power absorbed by energy storage device and equivalent admittance of static var compensation device.

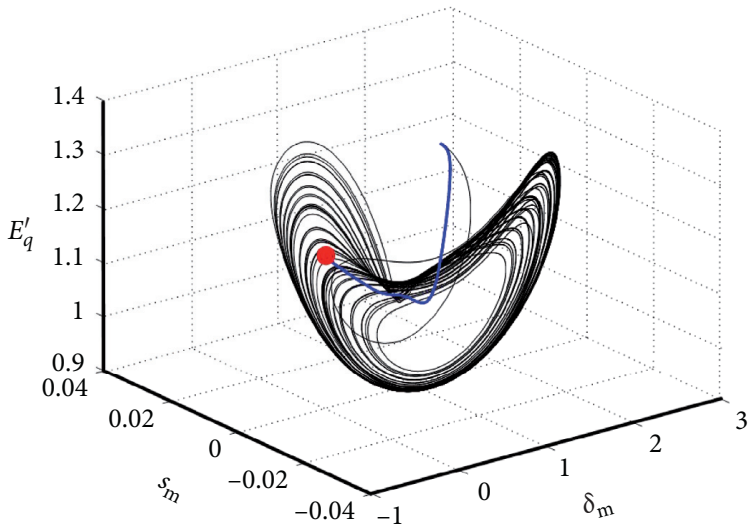

Chaotic attractor Evolution trajectory

__ Equilibrium fixed point

(a)

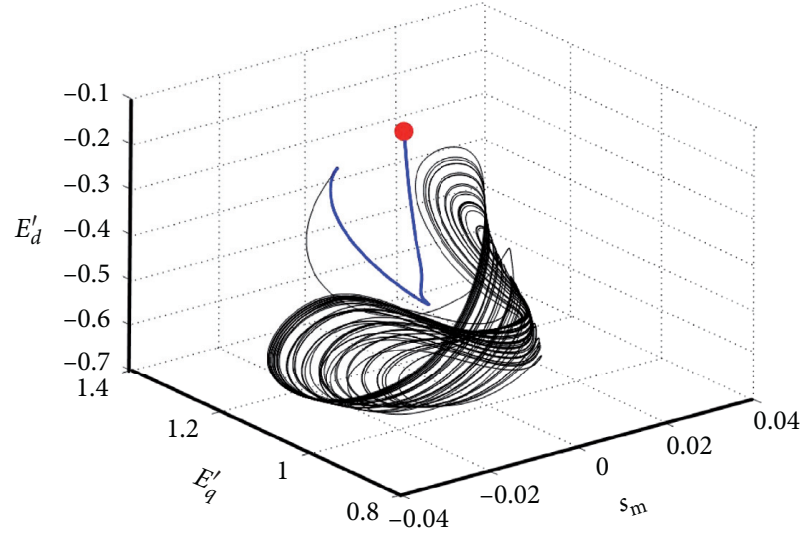

- Chaotic attractor

__ Evolution trajectory

_ Equilibrium fixed point

(b)

Figure 7: Continued. 


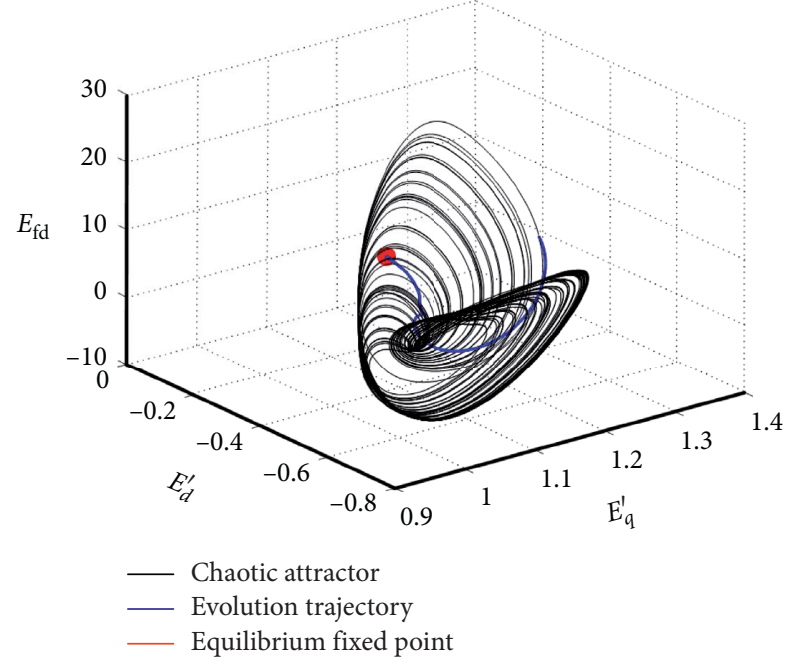

(c)

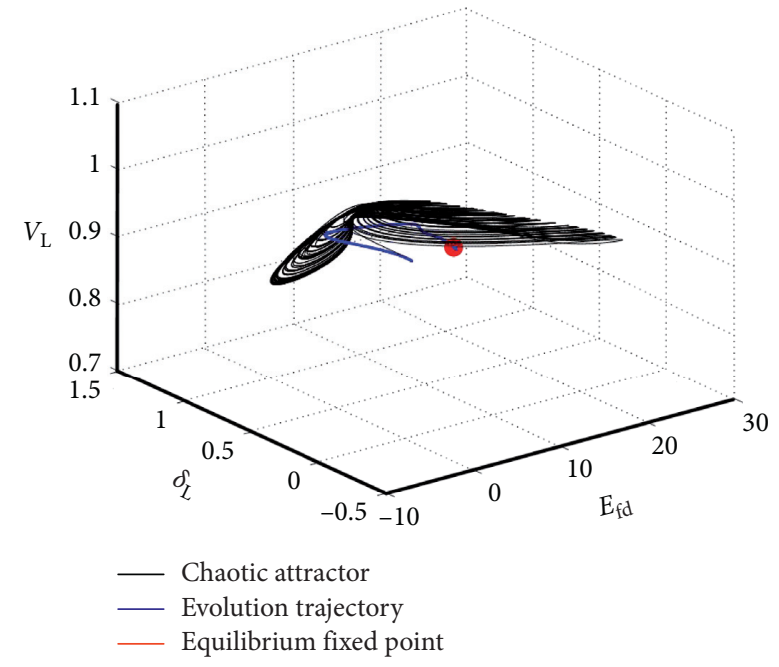

(e)

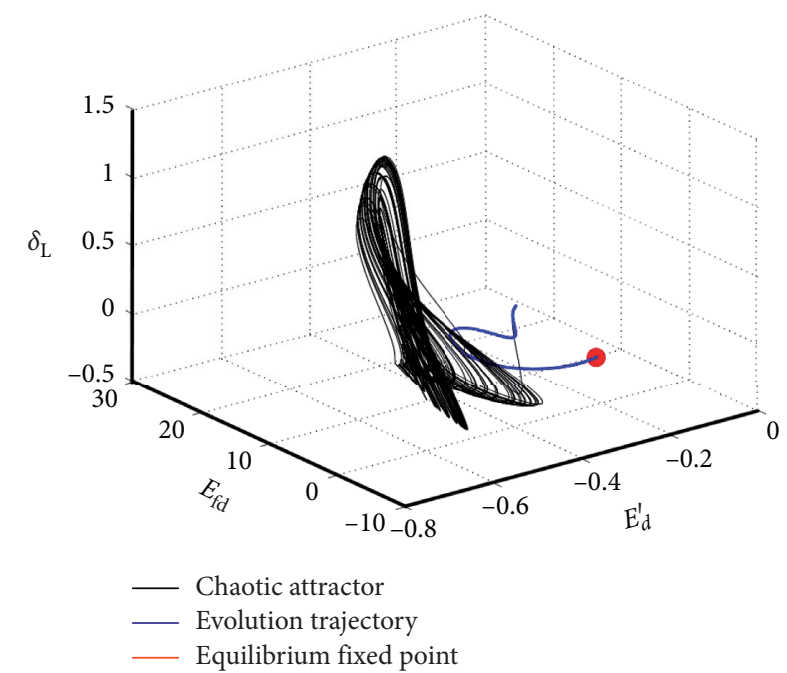

(d)

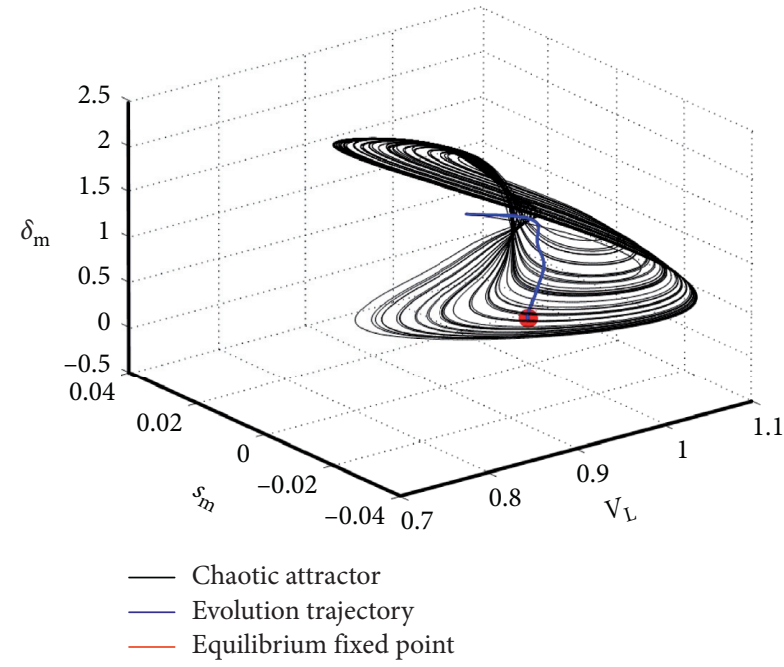

(f)

FIGURE 7: System state evolution process of uncontrolled and controlled power system in phase space. (a) $\delta_{\mathrm{m}}-s_{\mathrm{m}}-E_{\mathrm{q}}^{\prime}$ space. (b) $s_{\mathrm{m}}-$ $E_{\mathrm{q}}^{\prime}-E_{\mathrm{d}}^{\prime}$ space. (c) $E_{\mathrm{q}}^{\prime}-E_{\mathrm{d}}^{\prime}-E_{\mathrm{fd}}$ space. (d) $E_{\mathrm{d}}^{\prime}-E_{\mathrm{fd}}-\delta_{\mathrm{L}}$ space. (e) $E_{\mathrm{fd}}-\delta_{\mathrm{L}}-V_{\mathrm{L}}$ space. (f) $V_{\mathrm{L}}-s_{\mathrm{m}}-\delta_{\mathrm{m}}$ space.

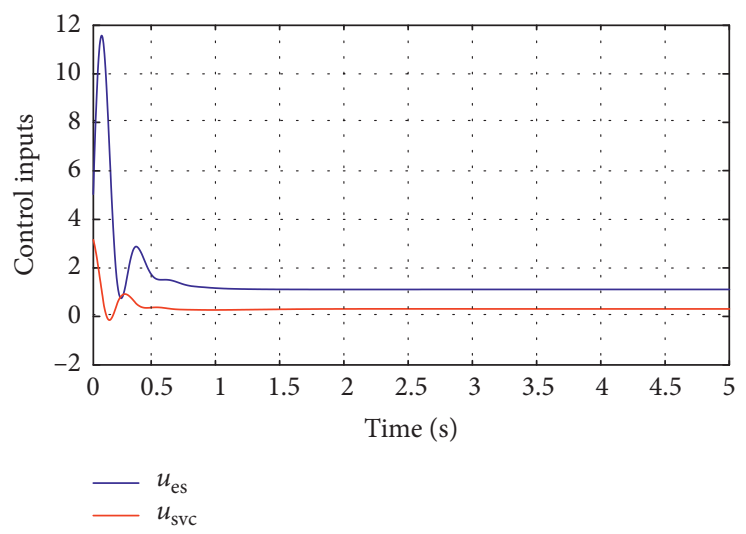

FIGURE 8: Control inputs for the seven-dimensional power system. 


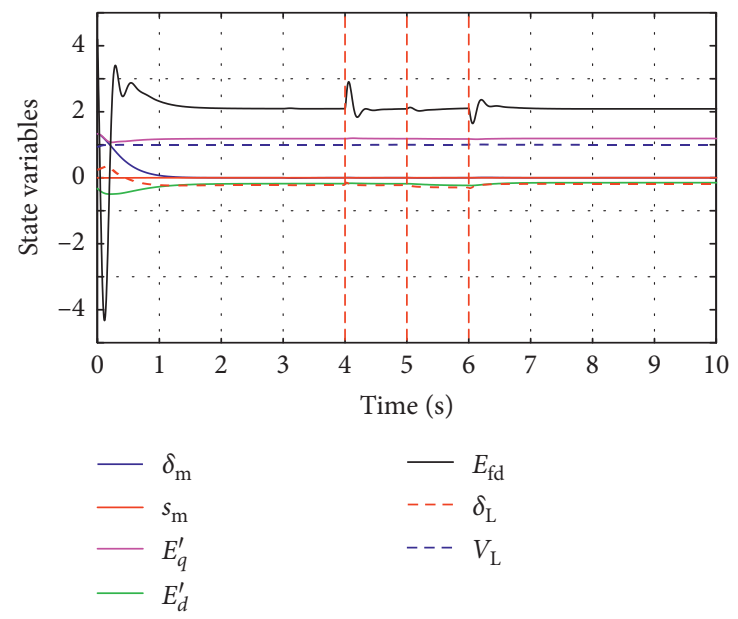

Figure 9: Time responses of controlled seven-dimensional power system considering parameter variation and uncertainty.

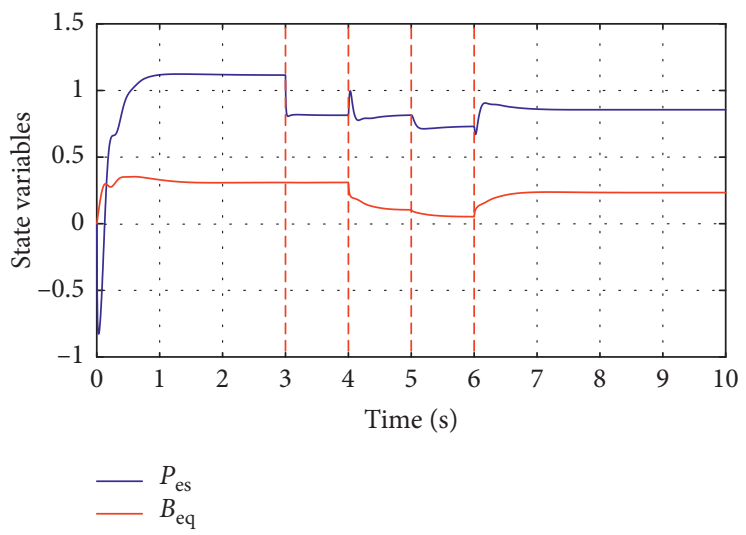

Figure 10: Time responses of active power absorbed by the energy storage device and equivalent admittance of static var compensation device considering parameter variation and uncertainty.

through a specific evolution trajectory. The time responses of designed control inputs (31) are shown in Figure 8 , and as it can be seen that control inputs are finally stable and smooth.

Example 2. Next, the robustness of proposed control method is verified. In system (23), parameter $d$ represents the damping coefficient of the power system; $P_{\mathrm{m}}$ represents mechanical input power of the generator; $Q_{1 \mathrm{~d}}$ represents reactive power of the load; and $P_{1 \mathrm{~d}}$ represents active power of the load. Since these parameters play an important role in the operation of power system, they are considered to verify the robustness of designed controller. Then consider the variation and uncertainty of these parameters (only $d$ is an uncertainty) as follows:

$$
\left\{\begin{array}{l}
d=0.05 \cdot[1+\sin (t)], \\
P_{\mathrm{m}}=1.363-0.3 \cdot \varepsilon(t-3), \\
Q_{1 \mathrm{~d}}=0+0.2 \cdot \varepsilon(t-4)-0.1 \cdot \varepsilon(t-6), \\
P_{1 \mathrm{~d}}=0+0.2 \cdot \varepsilon(t-5)-0.3 \cdot \varepsilon(t-6),
\end{array}\right.
$$

where $\varepsilon(t)$ is a step function.
In this way, the time responses of state variables of the controlled power system (24) can be obtained as shown in Figures 9 and 10. The two figures show that the power system can still recover from the original chaotic oscillation state to the equilibrium state under the condition of parameter variation and uncertainty, which proves that the proposed control method is robust to parameter variation and uncertainty.

\section{Conclusion}

Based on equivalence principle of control effect, an adaptive sliding mode control scheme is proposed for the controlled system. The unique advantage of the scheme is that no assumptions are introduced into the controller design process, and the designed controller has a wide range of applications. The proposed adaptive sliding mode controller scheme is used to control chaos in the seven-dimensional power system model, and the effectiveness and robustness of the designed controller are verified by simulation. In addition, the method proposed in the paper can be used to control other complex systems. Compared with controlling chaos in the two-dimensional and the four-dimensional power system models in 
other literature studies, this paper completes the chaos control problem in a complex seven-dimensional power system model. Compared with controllers designed in many literature studies which belong to abstract control inputs, this paper introduces dynamic models of the energy storage device and static var compensation device to make the designed controller more practical in engineering.

\section{Appendix}

Parameter $P_{\mathrm{g}}$ in system (23) represents active power transmitted by the generator and its expression is

$$
P_{\mathrm{g}}=E_{q}^{\prime} I_{q}+E_{d}^{\prime} I_{d}+\left(x_{d}^{\prime}-x_{q}^{\prime}\right) I_{d} I_{q}
$$

where

$$
\left\{\begin{array}{l}
I_{d}=\frac{\left[\left(\sin \phi-Y x_{q}^{\prime}\right) \cdot\left(Y E_{q}^{\prime}-a\right)+\cos \phi \cdot\left(Y E_{d}^{\prime}-b\right)\right]}{A} \\
I_{q}=\frac{\left[\cos \phi \cdot\left(Y E_{q}^{\prime}-a\right)-\left(\sin \phi-Y x_{d}^{\prime}\right) \cdot\left(Y E_{d}^{\prime}-b\right)\right]}{A} \\
A=\left[(\cos \phi)^{2}+\left(\sin \phi-Y x_{q}^{\prime}\right) \cdot\left(\sin \phi-Y x_{d}^{\prime}\right)\right] \\
a=E_{\mathrm{b}} Y_{3} \cos \left(\delta+\phi-\phi_{3}\right)+Y_{1} V_{\mathrm{L}} \cos \left(\delta_{\mathrm{L}}-\delta-\phi+\phi_{1}\right) \\
b=-E_{\mathrm{b}} Y_{3} \sin \left(\delta+\phi-\phi_{3}\right)+Y_{1} V_{\mathrm{L}} \sin \left(\delta_{\mathrm{L}}-\delta-\phi+\phi_{1}\right) \\
Y=\sqrt{\left(Y_{1} \cos \phi_{1}+Y_{3} \cos \phi_{3}\right)^{2}+\left(Y_{1} \sin \phi_{1}+Y_{3} \sin \phi_{3}\right)^{2}} \\
\phi=\arctan \left[\frac{\left(Y_{1} \sin \phi_{1}+Y_{3} \sin \phi_{3}\right)}{\left(Y_{1} \cos \phi_{1}+Y_{3} \cos \phi_{3}\right)}\right]
\end{array}\right.
$$

Parameters $P$ and $Q$ in system (23) represent active power and reactive power transmitted to the load bus, respectively, and their expressions are as follows:

$$
\left\{\begin{array}{l}
P=V_{\mathrm{t}} V_{\mathrm{L}} Y_{1} \cos \left(r_{1}\right)-V_{\mathrm{L}}^{2} Y_{1} \cos \left(\phi_{1}\right)+E_{\mathrm{b}} V_{\mathrm{L}} Y_{2} \cos \left(r_{2}\right)-V_{\mathrm{L}}^{2} Y_{2} \cos \left(\phi_{2}\right) \\
Q=V_{\mathrm{t}} V_{\mathrm{L}} Y_{1} \sin \left(r_{1}\right)+V_{\mathrm{L}}^{2} Y_{1} \sin \left(\phi_{1}\right)+E_{\mathrm{b}} V_{\mathrm{L}} Y_{2} \sin \left(r_{2}\right)+V_{\mathrm{L}}^{2} Y_{2} \sin \left(\phi_{2}\right),
\end{array}\right.
$$

where

$$
\left\{\begin{array}{l}
V_{\mathrm{t}}=\sqrt{V_{\mathrm{d}}^{2}+V_{\mathrm{q}}^{2}}, \\
V_{\mathrm{d}}=E_{d}^{\prime}-x_{q}^{\prime} I_{q}, \\
V_{\mathrm{q}}=E_{q}^{\prime}+x_{d}^{\prime} I_{d}, \\
r_{1}=\delta_{\mathrm{L}}-\left[\delta+\arctan \left(V_{\mathrm{d}} / V_{\mathrm{q}}\right)\right]-\phi_{1}, \\
r_{2}=\delta_{\mathrm{L}}-\phi_{2} .
\end{array}\right.
$$


Constant values of the above system parameters are

$$
\begin{aligned}
& Y_{1}=4.9752 \text {, } \\
& Y_{2}=1.6584 \text {, } \\
& Y_{3}=0 \text {, } \\
& \phi_{1}=-1.4711 \text {, } \\
& \phi_{2}=-1.4711 \text {, } \\
& \phi_{3}=-1.4711 \text {, } \\
& E_{\mathrm{b}}=1.0 \text {, } \\
& x_{d}=1.79 \text {, } \\
& x_{q}=1.71 \text {, } \\
& x_{d}^{\prime}=0.169 \text {, } \\
& x_{q}^{\prime}=0.23 \text {, } \\
& T_{d 0}=4.3 \text {, } \\
& T_{q 0}=0.85 \text {, } \\
& H=2.894 \text {, } \\
& \omega_{\mathrm{B}}=377 \text {, } \\
& d=0.05 \text {, } \\
& P_{0}=0.4 \text {, } \\
& Q_{0}=0.6 \text {, } \\
& B_{\mathrm{c}}=0.2 \text {, } \\
& p_{1}=0.24 \text {, } \\
& q_{1}=-0.02 \text {, } \\
& p_{2}=1.7 \text {, } \\
& q_{2}=-1.866, \\
& p_{3}=0.2 \text {, } \\
& q_{3}=1.6, \\
& Q_{1 \mathrm{~d}}=0 \text {, } \\
& P_{1 \mathrm{~d}}=0 \text {, } \\
& K_{\mathrm{A}}=200 \text {, } \\
& T_{\mathrm{A}}=0.05 \text {, } \\
& V_{\text {ref }}=1.12 \text {. }
\end{aligned}
$$

In these parameters, all values are in per unit values, except for angles, which are in degrees.

\section{Data Availability}

The data used to support the findings of this study are included within this article.

\section{Conflicts of Interest}

The authors declare no conflicts of interest in preparing this article.

\section{Acknowledgments}

This work was supported by the National Natural Science Foundation of China (Grant no. 51877162) and the Science Fund for Creative Research Groups of the National Natural Science Foundation of China (Grant no. 51521065).

\section{References}

[1] W. Ji and V. Venkatasubramanian, "Hard-limit induced chaos in a fundamental power system model," International Journal of Electrical Power \& Energy Systems, vol. 18, no. 5, pp. 279-295, 1996.

[2] V. Venkatasubramanian and W. Ji, "Coexistence of four different attractors in a fundamental power system model," IEEE Transactions on Circuits and Systems I: Fundamental Theory and Applications, vol. 46, no. 3, pp. 405-409, 1999.

[3] Z. Jing, D. Xu, Y. Chang, and L. Chen, "Bifurcations, chaos, and system collapse in a three node power system," International Journal of Electrical Power \& Energy Systems, vol. 25, no. 6, pp. 443-461, 2003.

[4] Y. Yu, H. Jia, P. Li, and J. Su, "Power system instability and chaos," Electric Power Systems Research, vol. 65, no. 3, pp. 187-195, 2003.

[5] M. L. Ma and F. H. Ming, "Bifurcation behavior and coexisting motions in a time-delayed power system," Chinese Physics B, vol. 24, no. 3, pp. 78-86, 2015.

[6] X. W. Chen, W. N. Zhang, and W. D. Zhang, "Chaotic and subharmonic oscillations of a nonlinear power system," IEEE Transactions on Circuits and Systems II-Express Briefs, vol. 52, no. 12, pp. 811-815, 2005.

[7] H.-D. Chiang, I. Dobson, R. J. Thomas, J. S. Thorp, and L. Fekih-Ahmed, "On voltage collapse in electric power systems," IEEE Transactions on Power Systems, vol. 5, no. 2, pp. 601-611, 1990.

[8] I. Dobson and H.-D. Chiang, "Towards a theory of voltage collapse in electric power systems," Systems \& Control Letters, vol. 13, no. 3, pp. 253-262, 1989.

[9] H. K. Chen, T. N. Lin, and J. H. Chen, "Dynamic analysis, controlling chaos and chaotification of a SMIB power system," Chaos Solitons \& Fractals, vol. 24, no. 3, pp. 1307-1315, 2005.

[10] D. Q. Wei and X. S. Luo, "Passivity-based adaptive control of chaotic oscillations in power system," Chaos, Solitons \& Fractals, vol. 31, no. 3, pp. 665-671, 2007.

[11] F. H. Min, M. L. Ma, Z. Wei, and E. R. Wang, "Chaotic control of the interconnected power system based on the relay characteristic function," Acta Physica Sinica, vol. 63, no. 5, pp. 1-8, 2014, in Chinese.

[12] J. Ni, C. Liu, K. Liu, and X. Pang, "Variable speed synergetic control for chaotic oscillation in power system," Nonlinear Dynamics, vol. 78, no. 1, pp. 681-690, 2014.

[13] C. Y. Ma, F. X. Wang, Z. J. Li et al., "Adaptive fixed-time fast terminal sliding mode control for chaotic oscillation in power system," Mathematical Problems in Engineering, vol. 2018, Article ID 5819428, 10 pages, 2018.

[14] H. Zhao, Y. J. Ma, S. J. Liu, S. G. Gao, and D. Zhong, "Controlling chaos in power system based on finite-time stability theory," Chinese Physics B, vol. 20, no. 12, pp. 1-8, 2011. 
[15] C.-Y. Ma, J.-H. Liu, and C.-L. Wang, "Chaos of a power system model and its control," Journal of Vibration and Control, vol. 18, no. 14, pp. 2176-2185, 2011.

[16] C. Wang, H. Zhang, W. Fan, and P. Ma, "Adaptive control method for chaotic power systems based on finite-time stability theory and passivity-based control approach," Chaos, Solitons \& Fractals, vol. 112, pp. 159-167, 2018.

[17] J. K. Ni, L. Liu, C. X. Liu, and X. Y. Hu, "Chattering-free time scale separation sliding mode control design with application to power system chaos suppression," Mathematical Problems in Engineering, vol. 2016, Article ID 5943934, 14 pages, 2016.

[18] J. B. Wang, C. X. Liu, Y. Wang, and G. C. Zheng, "Fixed time integral sliding mode controller and its application to the suppression of chaotic oscillation in power system," Chinese Physics B, vol. 27, no. 7, pp. 1-8, 2018.

[19] M. T. Alrifai and Z. Mohamed, "Sliding mode control of chaos in a single machine connected to an infinite bus power system," Mathematical Problems in Engineering, vol. 2018, Article ID 2703684, 13 pages, 2018.

[20] M. Zribi, M. T. Alrifai, and N. Smaoui, "Control of chaos in a single machine infinite bus power system using the discrete sliding mode control technique," Discrete Dynamics in Nature and Society, vol. 2018, Article ID 5758324, 14 pages, 2018.

[21] K. Rajagopal, A. Karthikeyan, P. Duraisamy, R. Weldegiorgis, and G. Tadesse, "Bifurcation, chaos and its control in a fractional order power system model with uncertainties," Asian Journal of Control, vol. 21, no. 1, pp. 184-193, 2019.

[22] J. Ni, L. Liu, C. Liu, X. Hu, and S. Li, "Fast fixed-time nonsingular terminal sliding mode control and its application to chaos suppression in power system," IEEE Transactions on Circuits and Systems II: Express Briefs, vol. 64, no. 2, pp. 151-155, 2017.

[23] J. Ni, L. Liu, C. Liu, X. Hu, and T. Shen, "Fixed-time dynamic surface high-order sliding mode control for chaotic oscillation in power system," Nonlinear Dynamics, vol. 86, no. 1, pp. 401-420, 2016.

[24] K. G. Rajesh and K. R. Padiyar, "Bifurcation analysis of a three node power system with detailed models," International Journal of Electrical Power \& Energy Systems, vol. 21, no. 5, pp. 375-393, 1999.

[25] H. J. Jia, Y. X. Yu, P. Li, and J. F. Su, "Relationship of power system chaos and instability modes," Proceedings of the CSEE, vol. 23, no. 2, pp. 1-4, 2003, in Chinese.

[26] Y. X. Yu, H. J. Jia, and C. S. Wang, "Chaotic phenomena and small signal stability region of electrical power systems," Science in China, vol. 44, no. 2, pp. 187-199, 2001.

[27] C. L. Li and S. M. Yu, "Adaptive chaotic control of permanent magnet synchronous motor," Acta Physica Sinica, vol. 60, no. 12 , pp. 1-7, 2011, in Chinese.

[28] C. Hua and X. Guan, "Adaptive control for chaotic systems," Chaos, Solitons \& Fractals, vol. 22, no. 1, pp. 55-60, 2004.

[29] J. K. Ni, L. Liu, C. X. Liu, X. Y. Hu, and A. A. Li, "Chaos suppression for a four-dimensional fundamental power system model using adaptive feedback control," Transactions of the Institute of Measurement and Control, vol. 39, no. 2, pp. 194-207, 2015.

[30] D. Q. Wei and X. S. Luo, "Adaptive robust control of chaotic oscillations in power system with excitation limits," Chinese Physics, vol. 16, no. 11, pp. 3244-3248, 2007.

[31] X. Shi and Z. Wang, "Robust chaos synchronization of fourdimensional energy resource system via adaptive feedback control," Nonlinear Dynamics, vol. 60, no. 4, pp. 631-637, 2010.

[32] X. Wang and Y. Wang, "Adaptive control for synchronization of a four-dimensional chaotic system via a single variable," Nonlinear Dynamics, vol. 65, no. 3, pp. 311-316, 2011.
[33] H. F. Cao and R. X. Zhang, "Adaptive synchronization of fractional-order chaotic system via sliding mode control," Acta Physica Sinica, vol. 60, no. 5, pp. 1-5, 2011, in Chinese.

[34] L. L. Huang and X. Qi, “The synchronization of fractional order chaotic systems with different orders based on adaptive sliding mode control," Acta Physica Sinica, vol. 62, no. 8, pp. 1-7, 2013, in Chinese.

[35] J. Ni, L. Liu, C. Liu, and J. Liu, "Fixed-time leader-following consensus for second-order multiagent systems with input delay," IEEE Transactions on Industrial Electronics, vol. 64, no. 11, pp. 8635-8646, 2017.

[36] M. P. Aghababa, "Finite-time chaos control and synchronization of fractional-order nonautonomous chaotic (hyperchaotic) systems using fractional nonsingular terminal sliding mode technique," Nonlinear Dynamics, vol. 69, no. 1-2, pp. 247-261, 2012.

[37] B. Wang, J. Ding, F. Wu, and D. Zhu, "Robust finite-time control of fractional-order nonlinear systems via frequency distributed model," Nonlinear Dynamics, vol. 85, no. 4, pp. 2133-2142, 2016.

[38] J. Ni, L. Liu, C. Liu, and X. Hu, "Fractional order fixed-time nonsingular terminal sliding mode synchronization and control of fractional order chaotic systems," Nonlinear Dynamics, vol. 89, no. 3, pp. 2065-2083, 2017.

[39] J. Fang, W. Yao, Z. Chen, J. Wen, and S. Cheng, "Design of anti-windup compensator for energy storage-based damping controller to enhance power system stability," IEEE Transactions on Power Systems, vol. 29, no. 3, pp. 1175-1185, 2014.

[40] D. P. Subramanian, R. P. K. Devi, and R. Saravanaselvan, “A new algorithm for analysis of SVC's impact on bifurcations, chaos and voltage collapse in power systems," International Journal of Electrical Power \& Energy Systems, vol. 33, no. 5, pp. 1194-1202, 2011.

[41] Y. Wang, H. Chen, and R. Zhou, "A nonlinear controller design for SVC to improve power system voltage stability," International Journal of Electrical Power \& Energy Systems, vol. 22, no. 7, pp. 463-470, 2000. 